\title{
Home Security Alarm Using Wemos D1 and HC- SR501 Sensor Based Telegram Notification
}

\author{
Refni Wahyuni ${ }^{1}$, Aditya Rickyta ${ }^{2}$, Uci Rahmalisa ${ }^{3}$, Yuda Irawan ${ }^{4 *}$ \\ ${ }^{1}$ Informatics Engineering, STMIK Hang TuahPekanbaru \\ ${ }^{2,3,4}$ Information Systems Department, STMIK Hang TuahPekanbaru \\ E-mail: refniabid@gmail.com ${ }^{1}$, adityarickyta@gmail.com ${ }^{2}$, ucirahmalisa89@gmail.com ${ }^{3}$, yudairawan89@gmail.com ${ }^{4}$ \\ *Corresponding Author
}

\begin{abstract}
Home Security Alarms in today's modern society only use CCTV that can only see the person without any notification that goes into the cellphone in dealing with the theft that occurred. To help the community in dealing with the theft that enters the house, a Home Security Alarm was made using WEMOS D1 and HC-SR501 Sensor with Telegram Notification. The whole tool is divided into several parts which consist of $\mathrm{HC}$ SR501 Sensor, WEMOS D1 and Buzzer. This tool works when the WEMOS D1 microcontroller processes the pear sensor as a motion detector and buzzer as a sound alarm if motion is detected, then the notification automatically enters into the Telegram Application, with this tool can monitor directly if anyone enters the house while being left
\end{abstract}

Keywords-Keywords: Wemos D1, Sensor HC-SR501, Telegram, CCTV, Buzzer.

\section{INTRODUCTION}

Seen from the point of view of the crime rate, it can be seen that the crime rate of finding empty houses is increasingly happening. The owner of the house does not know the condition of his house when traveling because there is no tool that can directly inform someone if the house has been entered by an unknown person and the ineffective information received when there is a suspicious movement at an unreasonable time.

Based on research from Muhammad Saleh et al, home is a place to store things valuable and perhaps very personal to the home owner because it is a lot of effort made by the community in order the house was spared from attempted theft. The venture is on among other things, conducting nightly patrols in the neighborhood community homes, put wild pets like the dog in the yard, padlocks the fence House.

Overcoming the problems mentioned earlier, many studies have been carried out, for example Design and Implementation of a WIFI Based Home Automation System, System supports a wide range of home automation devices like power management components, and security components.

The proposed system is better from the scalability and flexibility point of view than the commercially available home automation systems, Elshafee and Hamed [1]. Research conducted by Peby Wahyu Purnama and Yuni Rosita entitled Design of Smart Home System Using Nodemcu ESP8266 Communication-Based Telegram Messenger, where the final results of the test can be eliminated. Telegram Messenger application is very suitable for remote control and monitoring of Smart Home, Distance from 1, $7 \mathrm{~km}$ to $151 \mathrm{~km}$ of different areas obtained an average delay of 20.66 seconds [2]. Muhammad KhoiruSyabibi, ArkhanSubari in this article explained that when someone enters a house, he must press a button. If the input keypad is correct according to the passcode then the LED will turn off, indicating that the home security monitoring system is off for 10 seconds. If you enter the house without pressing the keypad according to the passcode then when the door is open the magnetic switch will be active, then the bell rings and the web indicator will change, indicating that he is a person who will commit a crime [3].

Hamzah \& Rusilawati research about NFC is a technology that can simplify and facilitate transactions, transfer data, and wireless connections between two communication devices that are very close to a few centimeters. This system is designed using an Android device that has NFC technology [4] research conducted by Yuda Irawan et al discusses detecting heart rate using a sensor device, researchers designed to measure heart rate as an alternative in maintaining health, using a pulse sensor and Arduino circuit. This research produces a heart rate application system as a control to monitor the condition of the patient's heart rate, can provide information about the heart rate using a heart rate sensor to patients and doctors who have an application to read the heart rate that has been made [5].

Research conducted by Joyner $\mathrm{R}$ et al made a system using the Sm630 fingerprint sensor as input to detect fingerprints from motorbike users. This system is also supported by the Arduino Uno kit with the ATmega328 microcontroller as the brain to process data from the fingerprint sensor to the LCD, motorbikes and alarms. There will only be five users who can access the motorbike and the system will turn on an alarm when an inappropriate fingerprint is attached to the sensor because the sensor will only communicate with the fingerprints stored in the sensor database [6].

Research conducted by Balakrishna Gokaraju is the implementation of a home security system in this study aimed at comparing the design and implementation of the program against ARM microprocessors and ATmega microprocessors [7]. Research conducted by G. M. Sultan Mahmud Rana et al is the design and implementation of home security systems that can save costs but are flexible [8]. Research conducted by Naser 
Abbas Hussein et al is a security door locking system based on Raspberry pi technology where cameras, keypads and pi-lids are used to provide an alarming system to notify homeowners, and to recognize people who come by giving them a user identity [9]. Long-distance home security alarm system using low power developed by applying WSN and GSM technology, namely the research of Huiping Huang and friends [10].

Next researchers propose a smart alarm system, which uses machine learning techniques to intelligently detect threats using environmental data that has been collected by Arduino sensors and microcontrollers after which it notifies homeowners via email in real time [11]. Subsequent research discusses the Smart Home Security Monitor System Internet of Things (IoT) and Wireless Sensor Networks (WSNs) this monitoring system provides checks for events that occur and sends alarm messages to the user. This system uses three gradations, namely Client Sensor, Data Collector and Data Center [12].

Further research is the home security system by sending notifications to home owners using the Internet if someone enters secretly and can hide the alarm if needed by the home owner [13]. Further research is a system that can recognize security alarm events obtained from sensor nodes and forwarded by means of hop-by-hop transmission [14]. The next researcher uses the Internet of Things (IoT) for smart home security which can decide which people can enter the house and who cannot use cellphones and web applications [15]. Research by Chan Zhen Yue et al, namely the design and implementation of smart homes using sound [16]. The IoT project this time is focused on a smart wireless home security system which will send a warning to home owners using the Internet in the event of a breach which will optionally raise the alarm [17].

The next research states that the concept of smart homes and smart cities is in accordance with the state program "Russian Digital Economy", so it is advisable to use technology related to state programs [18]. In subsequent research using RFID, the terminal can precisely change the alarm mode and notify the home owner of an intruder quickly [19]. A home security system and teleguard that gives a warning when the owner is at home but also provides a voice message that comes from a previously recorded voice via a cellphone so that the home owner can control the condition of his house [20]. A home security system or what is called a smart home is needed nowadays and there are also very many enthusiasts because it is very helpful for home owners when the owner is far away or is outside the house [21].

There is also a study that discusses the safety of the home using Bluetooth using a microcontroller Peripheral Interface Controller (PIC) (16F1937) as the main manager and the equipment is connected to the microcontroller peripheral port via a relay [22]. This study discusses an approach to combining speech recognition technology with the Internet of Things (IoT) for smart homes [23]. The next research discusses a smart home which is how it works, namely when the owner leaves the house and it turns out that other people who come to the house will send an SMS to the home owner / user and the alarm will go off. after the alarm is on, the recorder will automatically record which results will be visible to the owner / user [24]. the system can recognize events and an alarm will fire using sensor nodes and forwarded by means of hop-by-hop transmission. There is a sensor network, a mobile home security robot (HSMR) and a home server in this system [25]. Homes without a sophisticated security system have a higher chance of being targeted by criminals than homes that use sophisticated security systems [26]. Subsequent research proposes a ZigBee and PSTN based wireless smart home sensor network system using the PIC18LF4620 SCM and 2.4G RF transceiver modules [27]. Subsequent research proposes algorithms to detect intruders into the room. The main idea of the proposed method is to estimate the feature variation of the room acoustic transfer function (RTF) [28]. In making home security systems, many use the Internet of Things framework which is popular in the Security Sector [29].

IoT devices are an integral part of IoT-based systems [30]. From some of the previous studies above, it can be concluded that the use of telegram is still very minimal, so that researchers are interested in using telegram as a medium for conveying home security information. This Telegram application will later be used as a medium for system monitoring activities to be made. This Telegram application will be used as a medium for system monitoring activities to be made. Telegram itself was chosen because this application is free, with a fairly complete and growing Bot API. The existence of the Application Programming Interface (API) feature is one of the advantages of this application. This application has a bot that functions as a service to create a computational system that will be created, and can also be developed by the wider community. Smartphones equipped with Android OS can be used to monitor the home security system and detect someone's presence by using a motion sensor such as the PIR sensor which detects the wavelength of passive infrared rays produced by the human body and then makes a sound from the buzzer device and will provide notification via Telegram application.

Based on the description above, a research is submitted for research with the title "Home Security Alarm Using Wemos D1 and Sensor HC-SR501 With Telegram Notification".

\section{METHODS}

Researchers use the prototype method, because this method is a method that is widely used by software developers. The essence of this method is the work of a model development into a final system. The following are the stages of making a prototype method, namely:

\section{Collection of Needs}

Researchers analyze the needs in the design of the control system to be built. Among them is the selection of the required hardware and software. In this case the researchers used the Wemos D1 microcontroller as the main hardware and Telegram as an intermediary for communication between the smartphone and hardware.

\section{Building Prototyping}

In this case the researcher describes the input and output formats that will be produced by the security system being 
created. Here the researcher describes the input and output schemes that will be received by Wemos D1.

\section{Prototype Evaluation}

Furthermore, after the prototyping development stage, the researcher defines the format and requirements of the entire security system, identifies all the requirements, and an outline of the system to be built, for example the communication path between the Wemos D1 microcontroller and the Telegram platform.

\section{Encoding the System}

In this stage the prototyping that has been agreed upon is translated into an appropriate programming language as a process for inputting commands that will be received on the microcontroller. In this system, the programming language used is $\mathrm{C}++$ using the Arduino IDE software.

\section{Testing the System}

In this stage, the modules that have been made are combined and tested to find out whether the system that has been built is in accordance with the design and whether there are still errors or not. Testing using android is testing carried out directly by the user.

\section{System Evaluation}

System evaluation is not prototyping evaluation, system evaluation is evaluating the finished system or software and hardware whether it is in accordance with the wishes or not. If not, then the system will be revised again and return to stages 4 and 5. If the system is said to be OK, the system is ready to continue at the next stage.

\section{Using the System}

This stage is the final stage of creating a security system using the Prototyping Model method. At this stage, the finished tools that have passed the test are ready for use.

\section{IMPLEMENTATION}

Implementation is one of the stages in system development, where this stage is the stage of placing a home security alarm at home so that it is ready for operation and can be seen as an effort to realize the system that has been designed.

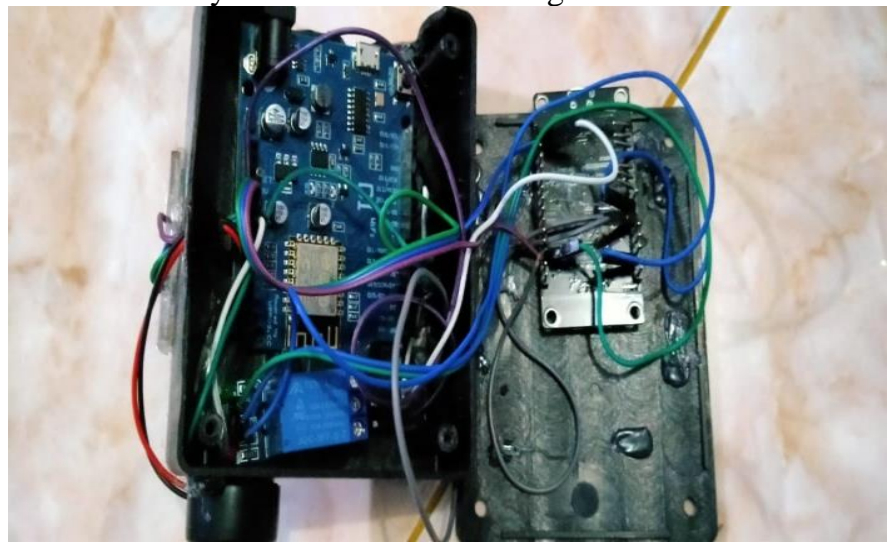

Fig. 1 Home Security Alarm Using Wemos D1 and Sensor HC-SR501

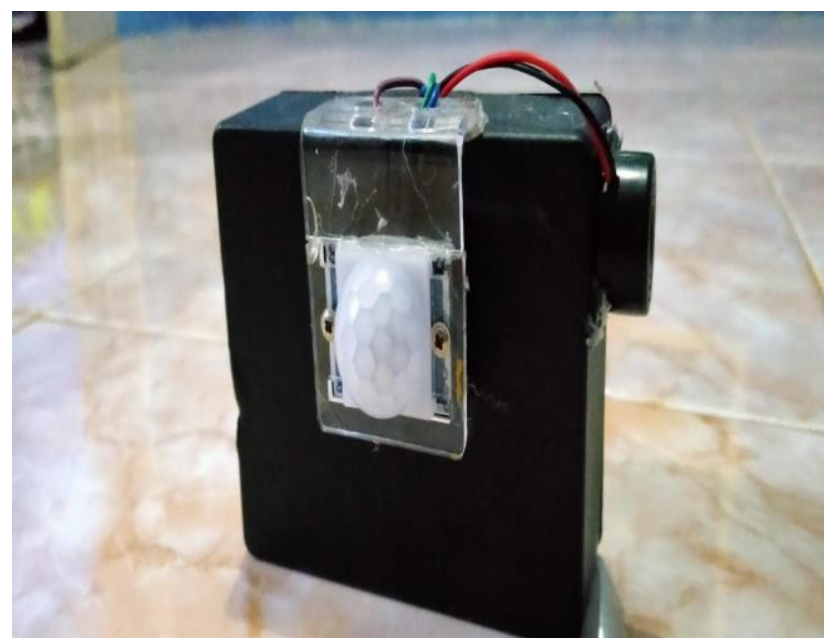

Fig. 2. Home Security Alarm

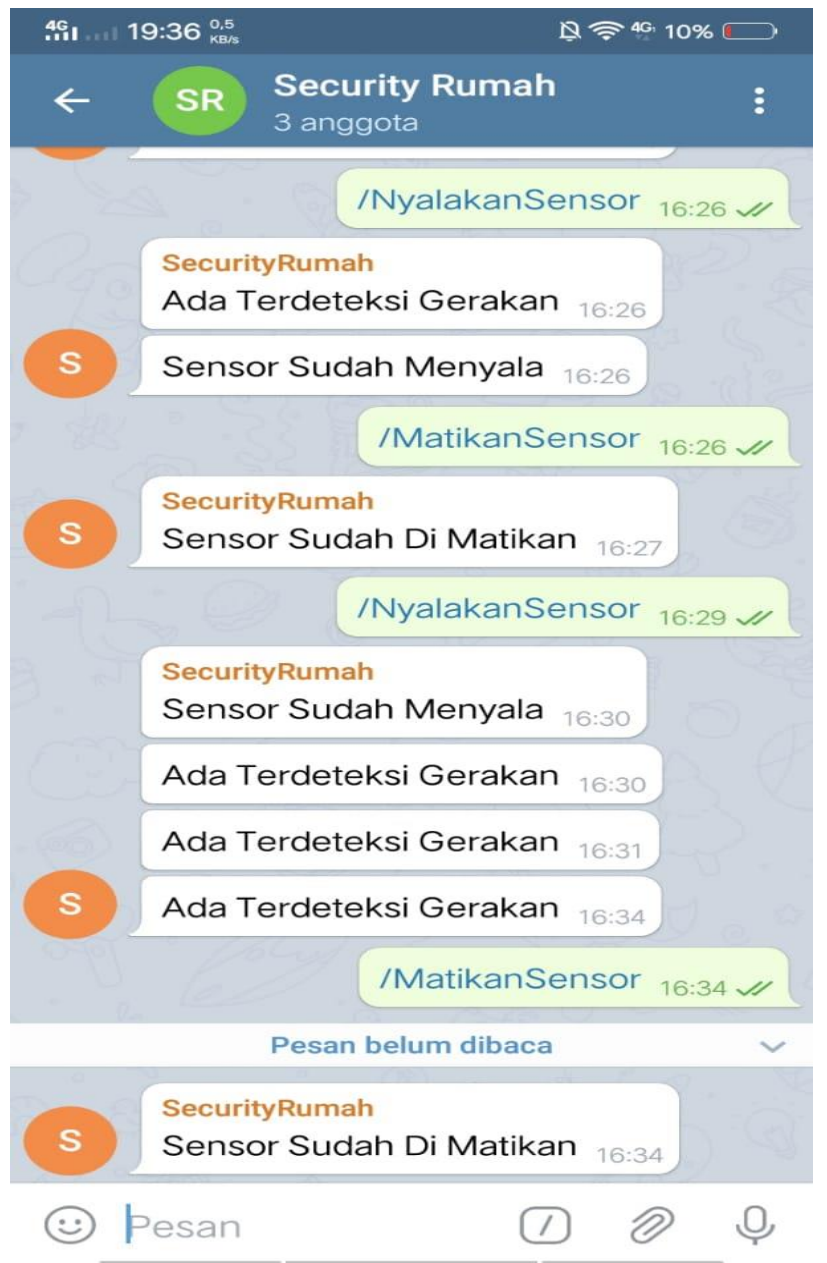

Fig. 3. Telegram Notification Display

\section{A. System Testing}

Testing the WEMOS D1 system on Home Alarm Security can be done with the following steps:

1. Connect the cellphone charger cable to the Home Security Alarm hardware circuit and press the on button. 
2. After that open the cellphone and the telegram application, then enter the command / Turn on Sensor to turn on the device to connect to the telegram.

3. If successful, the tool will automatically activate and detect every movement that crosses the sensor of the pear causing a sound on the buzzer and then giving a notification to the telegram.

4. To check the status of whether the tool is turned off or on, type / StatusSensor then we will be able to see whether the tool is turned off or on.

5. When finished, type the command / Turn off the sensor, the sensor will automatically turn off and cannot detect human beings who cross it.

\section{B. Testing Systems on the Prototype}

Tests are carried out to test the system on a prototype designed with the movement of humans passing through it. The result is shown Table 1.

Table I. Home Alarm Security Testing

\begin{tabular}{|c|c|c|c|}
\hline Test & Sensor & Buzzer & Result \\
\hline & On & On & $\begin{array}{c}\text { Can detect people who enter the } \\
\text { house and send notifications to } \\
\text { telegram }\end{array}$ \\
\hline
\end{tabular}

\section{PIR Sensor Testing}

Testing by testing the distance on the pear sensor. The distance on the sensor is generally $5 \mathrm{~m}$, so the device sounds and can detect human presence and provide message notifications on telegram.

\section{CONCLUSION}

Based on the results of the analysis, design and implementation that has been done. Then several conclusions can be drawn including the following:

1. Home Security Alarm uses a PIR Sensor as a detection of the presence of humans entering the house, a Buzzer which provides notification sounds inside the house, and a telegram which is a software to receive and activate notifications if someone is detected entering the house.

2. Home Security Wemos d1 alarm and pir sensor can more effectively assist people in overcoming theft that occurs at home.

\section{REFERENCES}

[1] ElShafee, A., \& Hamed, K. A. (2012). Design and implementation of a WIFI based home automation system. World academy of science, engineering and technology, 68, 2177-2180.

[2] Purnawan, P. W., \& Rosita, Y. (2019). Design and Build a Smart Home System Using NodeMCU Esp8266 Communication Based Telegram Messenger. Techno. Com, 18(4), 348-360.

[3] Syabibi, M. K., \&Subari, A. (2016). Design and Build a WEB-Based Home Security Monitoring System Using Raspberry PI B + as Server and Control Media. Echo of Technology, 19(1), 22-29.
[4] Hamzah, M. L., Desnelita, Y., Purwati, A. A., Rusilawati, E., Kasman, R., \& Rizal, F. (2019). A review of Near Field Communication technology in several areas. Revista ESPACIOS, 40(32).

[5] Irawan, Y., Fernando, Y., \&Wahyuni, R. (2019). Detecting Heart Rate Using Pulse Sensor As Alternative Knowing Heart Condition. Journal of Applied Engineering and Technological Science (JAETS), 1(1), 30-42.

[6] Oroh, J. R., Kendekallo, E., Sompie, S. R., \&Wuwung, J. O. (2014). Rancangbangunsistemkeamanan motor denganpengenalansidikjari. Jurnal Teknik elektro dan komputer, 3(1), 36-42.

[7] B. Gokaraju, D. Yessick, J. Steel, D. A. Doss and A. C. Turlapaty, "Integration of intrusion detection and web service alarm for home automation system using 'ARM' microprocessor," SoutheastCon 2016, Norfolk, VA, 2016, pp. 1-2, doi: 10.1109/SECON.2016.7506717.

[8] G. M. S. Mahmud Rana, A. A. Mamun Khan, M. N. Hoque and A. F. Mitul, "Design and implementation of a GSM based remote home security and appliance control system," 2013 2nd International Conference on Advances in Electrical Engineering (ICAEE), Dhaka, 2013, pp. 291-295, doi: 10.1109/ICAEE.2013.6750350.

[9] N. A. Hussein and I. Al Mansoori, "Smart Door System for Home Security Using Raspberry pi3," 2017 International Conference on Computer and Applications (ICCA), Doha, 2017, pp. 395-399, doi: 10.1109/COMAPP.2017.8079785.

[10] H. Huang, S. Xiao, X. Meng and Y. Xiong, "A Remote Home Security System Based on Wireless Sensor Network and GSM Technology," 2010 Second International Conference on Networks Security, Wireless Communications and Trusted Computing, Wuhan, Hubei, 2010, pp. 535538, doi: 10.1109/NSWCTC.2010.132.

[11] A. E. Kashef and N. Barakat, "Intelligent Alarm System to Protect Small, Valuable Items," 2018 International Conference on Computer and Applications (ICCA), Beirut, 2018, pp. 326-330, doi: 10.1109/COMAPP.2018.8460470.

[12] X. Hong, C. Yang and C. Rong, "Smart Home Security Monitor System," 2016 15th International Symposium on Parallel and Distributed Computing (ISPDC), Fuzhou, 2016, pp. 247-251, doi: 10.1109/ISPDC.2016.42.

[13] S. Somani, P. Solunke, S. Oke, P. Medhi and P. P. Laturkar, "IoT Based Smart Security and Home Automation," 2018 Fourth International Conference on Computing Communication Control and Automation (ICCUBEA), Pune, India, 2018, pp. 1-4, doi: 10.1109/ICCUBEA.2018.8697610.

[14] Y. Kim, H. Kim, S. Lee and K. Lee, "Ubiquitous Home Security Robot Based on Sensor Network," 2006 IEEE/WIC/ACM International Conference on Intelligent Agent Technology, Hong Kong, 2006, pp. 700704, doi: 10.1109/IAT.2006.128.

[15] S. Pawar, V. Kithani, S. Ahuja and S. Sahu, "Smart Home Security Using IoT and Face Recognition," 2018 Fourth International Conference on Computing Communication Control and Automation (ICCUBEA), Pune, India, 2018, pp. 1-6, doi: 10.1109/ICCUBEA.2018.8697695.

[16] C. Z. Yue and S. Ping, "Voice activated smart home design and implementation," 2017 2nd International Conference on Frontiers of Sensors Technologies (ICFST), Shenzhen, 2017, pp. 489-492, doi: 10.1109/ICFST.2017.8210563.

[17] R. K. Kodali, V. Jain, S. Bose and L. Boppana, "IoT based smart security and home automation system," 2016 International Conference on Computing, Communication and Automation (ICCCA), Noida, 2016, pp. 1286-1289, doi: 10.1109/CCAA.2016.7813916.

[18] V. Zhmud, A. Liapidevskiy, H. Roth and J. Nosek, "The Concept of a Smart Home: Security, Additional Features and Augmented Reality," 2019 International Multi-Conference on Industrial Engineering and Modern Technologies (FarEastCon), Vladivostok, Russia, 2019, pp. 1-8, doi: 10.1109/FarEastCon.2019.8933841.

[19] M. Fujikawa, H. Doi and S. Tsujii, "Proposal for a New Home Security System in terms of User-friendliness and Prompt Intrusion Notification," 2006 IEEE International Conference on Computational Intelligence for Homeland Security and Personal Safety, Alexandria, VA, 2006, pp. 6166, doi: 10.1109/CIHSPS.2006.313308.

[20] R. K. Maurya, A. K. Singh, B. K. Kanaujia, B. D. Yadav and R. Rani, "Wireless home security \& teleguard system," 2008 IET International Conference on Wireless, Mobile and Multimedia Networks, Beijing, 2008, pp. 78-80, doi: 10.1049/cp:20080148. 
[21] Q. I. Sarhan, "Arduino Based Smart Home Warning System," 2020 IEEE 6th International Conference on Control Science and Systems Engineering (ICCSSE), Beijing, China, 2020, pp. 201-206, doi: 10.1109/ICCSSE50399.2020.9171939.

[22] S. Zondo, K. Ogudo and P. Umenne, "Design of a Smart Home System Using Bluetooth Protocol," 2020 International Conference on Artificial Intelligence, Big Data, Computing and Data Communication Systems (icABCD), Durban, South Africa, 2020, pp. 1-5, doi: 10.1109/icABCD49160.2020.9183815.

[23] P. Wang, X. Lu, H. Sun and W. Lv, "Application of speech recognition technology in IoT smart home," 2019 IEEE 3rd Advanced Information Management, Communicates, Electronic and Automation Control Conference (IMCEC), Chongqing, China, 2019, pp. 1264-1267, doi: 10.1109/IMCEC46724.2019.8984175.

[24] M. S. Sefat, A. A. M. Khan and M. Shahjahan, "Implementation of vision based intelligent home automation and security system," 2014 International Conference on Informatics, Electronics \& Vision (ICIEV), Dhaka, 2014, pp. 1-6, doi: 10.1109/ICIEV.2014.6850818.

[25] R. K. Kodali, S. C. Rajanarayanan, A. Koganti and L. Boppana, "IoT based security system," TENCON 2019 - 2019 IEEE Region 10 Conference (TENCON), Kochi, India, 2019, pp. 1253-1257, doi: 10.1109/TENCON.2019.8929420
[26] I. C. P. Mendoza, S. M. Timbol, M. J. C. Samonte and E. B. Blancaflor, "ImHome: An IoT for Smart Home Appliances," 2020 IEEE 7th International Conference on Industrial Engineering and Applications (ICIEA), Bangkok, Thailand, 2020, pp. 761-765, doi: 10.1109/ICIEA49774.2020.9101906.

[27] Lili Liang, Lianfen Huang, Xueyuan Jiang and Yan Yao, "Design and implementation of wireless Smart-home sensor network based on ZigBee protocol," 2008 International Conference on Communications, Circuits and Systems, Fujian, 2008, pp. 434-438, doi: 10.1109/ICCCAS.2008.4657808.

[28] Young-Keun Choi, Ki-Man Kim, Ji-Won Jung, Seung-Yong Chun and Kyu-Sik Park, "Acoustic intruder detection system for home security," in IEEE Transactions on Consumer Electronics, vol. 51, no. 1, pp. 130138, Feb. 2005, doi: 10.1109/TCE.2005.1405710.

[29] R. G. Anvekar and R. M. Banakar, "IoT application development: Home security system," 2017 IEEE Technological Innovations in ICT for Agriculture and Rural Development (TIAR), Chennai, 2017, pp. 68-72, doi: 10.1109/TIAR.2017.8273688.

[30] R. G. Anvekar, R. M. Banakar and R. R. Bhat, "Design alternatives for end user communication in IoT based system model," 2017 IEEE Technological Innovations in ICT for Agriculture and Rural Development (TIAR), Chennai, 2017, pp. 121-125, doi: 10.1109/TIAR.2017.827369. 